\title{
Characterization of ionization injection in gas mixtures irradiated by subpetawatt class laser pulses
}

\author{
A. Zhidkov, ${ }^{1,2,3}$ N. Pathak, ${ }^{1,2, *}$ J. K. Koga $\odot,{ }^{3}$ K. Huang $\odot,{ }^{2,3}$ M. Kando, ${ }^{2,3}$ and T. Hosokai ${ }^{1,2}$ \\ ${ }^{1}$ Institute of Scientific and Industrial Research, Osaka University, Mihogaoka 8-1, Ibaraki, Osaka 567-0047, Japan \\ ${ }^{2}$ Laser Accelerator R\&D, Innovative Light Sources Division, RIKEN SPring-8 Center, Kouto 1-1-1, Sayo-cho, \\ Sayo-gun, Hyogo 679-5148, Japan \\ ${ }^{3}$ Kansai Photon Science Institute, National Institutes for Quantum and Radiological Science and Technology, \\ Kizugawa, Kyoto 619-0215, Japan
}

(Received 2 September 2019; accepted 31 January 2020; published 26 February 2020)

\begin{abstract}
The effects of ionization injection in low- and high- $Z$ gas mixtures for the laser wake field acceleration of electrons are analyzed with the use of balance equations and particle-in-cell simulations via test probe particle trajectories in realistic plasma fields and direct simulations of charge loading during the ionization process. It is shown that electrons appearing at the maximum of the laser pulse field after optical ionization are trapped in the first bucket of the laser pulse wake. Electrons, which are produced by optical field ionization at the front of laser pulse, propagate backward; some of them are trapped in the second bucket, the third bucket, and so on. The efficiency of ionization injection is not high, several $\mathrm{pC} / \mathrm{mm}$ per bucket. This injection becomes competitive with wave breaking injection at lower plasma density and over a rather narrow range of laser pulse intensity.
\end{abstract}

DOI: 10.1103/PhysRevResearch.2.013216

\section{INTRODUCTION}

Interest in laser wake field acceleration (LWFA) of electrons [1] has rapidly grown over recent decades owing to notable results achieved by several groups [2-7]. The production of $8-\mathrm{GeV}$ electron bunches, in plasma channels a few tens of centimeters long, with a charge around several $\mathrm{pC}$ [7], demonstrates that LWFA may become a valuable branch in the electron accelerator family. Electron self-injection in the acceleration part of the laser wake field is a fundamental process for single-stage electron laser wake field acceleration [8-10] as well as for a plasma cathode in multistage LWFA $[11,12]$. Presently, electron self-injection is considered to be the only way to generate electron bunches with characteristics suitable for their further acceleration in laser pulse wakes $[8,12]$. Therefore, investigation of the various mechanisms of electron self-injection is particularly important in the development of staging in LWFA.

So far plasma wave breaking mechanisms have been considered as the primary source of electron self-injection. Several mechanisms such as wave breaking at density ramps $[8,10]$, parametric resonances [13], frequency chirp $[14,15]$, relativistic wave breaking $[16,17]$, and wave breaking provoked by external sources [18-20] have been proposed and studied both theoretically and experimentally. Recently, another mechanism of electron self-injection has been proposed,

\footnotetext{
*naveenpathak@sanken.osaka-u.ac.jp

Published by the American Physical Society under the terms of the Creative Commons Attribution 4.0 International license. Further distribution of this work must maintain attribution to the author(s) and the published article's title, journal citation, and DOI.
}

ionization injection [21-35], which essentially differs from the common wave breaking process.

Ionization injection should occur in a low- $Z$ gas with a high- $Z$ dope, for example, $\mathrm{He}-\mathrm{N}_{2}$ and $\mathrm{He}-\mathrm{Ne}$. Optical field ionization of inner shell electrons of the high- $Z$ dope in the vicinity of the maximum of the laser pulse field produces a number of low-energy electrons moving with a phase different from that of the laser pulse wake [21]. These electrons can be trapped and further accelerated.

Separation of ionization injection from the wave breaking injection can be done in rather low-density plasma, which requires high-power laser pulses in order to reach the selffocusing regime for essential electron acceleration. A laser pulse should have power equal to 2-3 times the critical power for self-focusing, $P_{\mathrm{cr}}=1.7 \times 10^{-2} N_{\mathrm{cr}} / N_{e}$ (TW) [36], where $N_{e}$ is the plasma density and $N_{\mathrm{cr}}$ is the critical density corresponding to the laser wavelength. This implies that for $N_{e}<10^{18} \mathrm{~cm}^{-3}$ and $\lambda=0.8 \mu \mathrm{m}$, laser pulses with powers of the order of the petawatt class are required. In higher-density plasma wave breaking processes [8-20] become essential and therefore mess up the picture of ionization injection. Moreover, various instabilities of laser pulse propagation such as hosing, filamentation, and over-self-focusing [10] surely make the ionization injection process impractical. As will be shown here, the requirement for the laser pulse duration also becomes too strong.

The principle of ionization injection and acceleration in a running wake wave can be illustrated by the simplest field structure consisting of a constant electric field with a negative strength $-E$, lasting from $V_{g} t$ to $L+V_{g} t$, where $t$ is the time and $L$ is the length of the wave moving with velocity $V_{g}$. If the initial position of an electron in this field at $t=0$ is $x=0$ (rear side of the wave), the electron, in order to be further accelerated or to move with the wave, must have a velocity $v_{e}$ such that $v_{e}>V_{g}$. This is the typical condition for the 


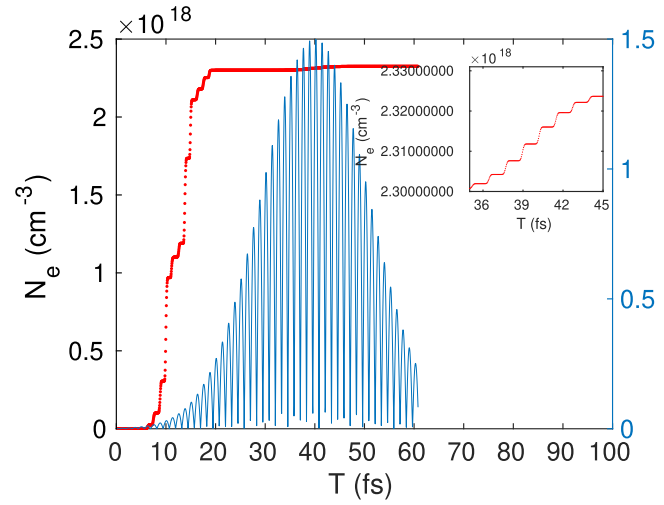

(a)

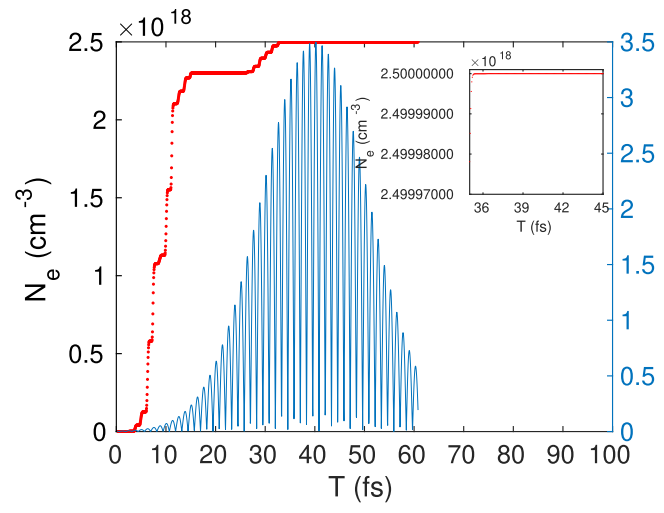

(c)

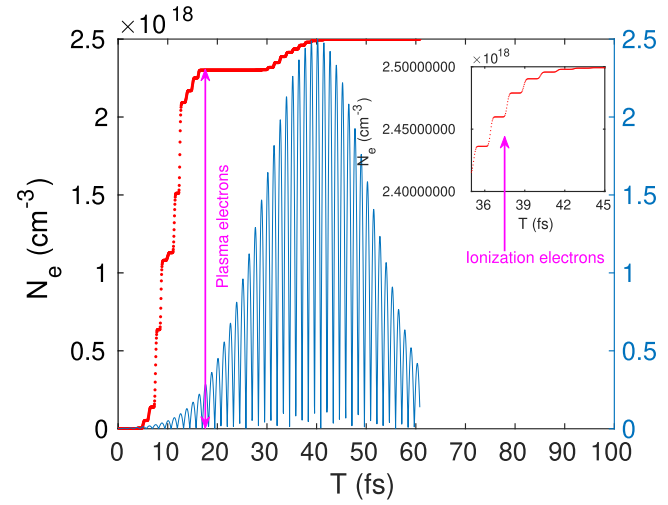

(b)

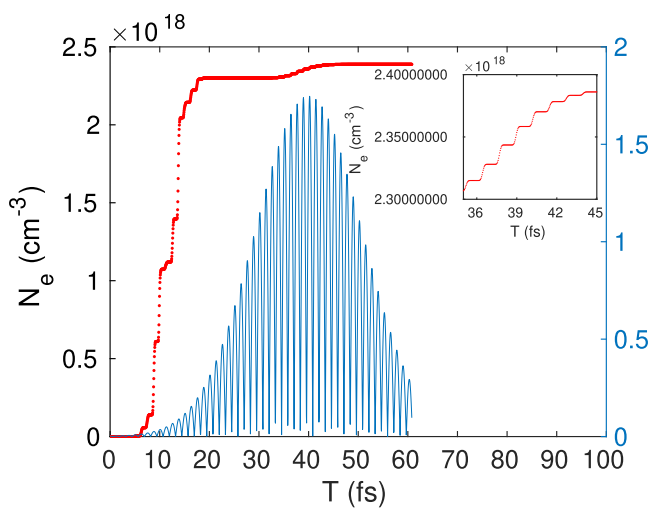

(d)

FIG. 1. Dynamics of the electron density in the gas mixture $\mathrm{He}-\mathrm{N}_{2}$ with $5 \%$ of $\mathrm{N}_{2}$ (a)-(c) along the laser axis and (d) off-axis, depending on the laser pulse intensity (a) $a_{0 \max }=1.5$, (b) $a_{0 \max }=2.5$, and (c) and (d) $a_{0 \max }=3.5$. Arrows in (b) shows the range of plasma electrons and ionization electrons. Insets show the electron densities around the peak of the laser pulse.

wave breaking process. In contrast, if an electron at $t=0$ has position $x=L$ (front of the wave), it can be trapped even if its velocity is initially zero. For this to happen the time $t_{0}$, over which the electron velocity becomes equal to the group velocity of the wave, should be such that the electron position must still exceed the position of the wave rear $x_{0}=V_{g} t_{0}$,

$$
\begin{aligned}
V_{g} & =\frac{p_{0}+e E t_{0}}{m c \sqrt{1+\left(\frac{p_{0}+e E t_{0}}{m c}\right)^{2}},} \\
V_{g} t_{0} & \leqslant L+\frac{m c^{2}}{e E}\left[\sqrt{1+\left(\frac{p_{0}+e E t_{0}}{m c}\right)^{2}}-1\right],
\end{aligned}
$$

where $p_{0}$ is the initial electron momentum. The solution of Eq. (1) gives a rather soft condition $U \geqslant m c^{2}\left(1-\frac{1}{\gamma_{g}}\right)-p_{0} V_{g}$, where $U=e E L$ is the potential difference and $\gamma_{g}$ is the relativistic factor for $V_{g}$. The analysis can be done for a running wave with nonlinear field strength like $E(t)=\alpha^{(n)}\left(x-V_{g} t\right)^{n}$ in integrated form as well, where $\alpha^{(n)}$ is a constant. Numerically, the results are qualitatively similar but the analysis takes much more space since it is not as clear as the constant field one.

In particular for a laser pulse wake, assuming $E=E_{L} \frac{\omega_{\mathrm{pl}}}{\omega_{0}}$ [36], $p_{0}=0$, and $\gamma_{g} \gg 1$, where $E_{L}$ is the strength of the laser pulse, $\omega_{\mathrm{pl}}$ the plasma frequency, and $\omega_{0}$ the laser frequency, one can get the requirement for the trapping length $L>\frac{\lambda_{\mathrm{pl}}}{2 \pi a_{0}}$, with $\lambda_{\mathrm{pl}}$ the plasma wavelength and $a_{0}=\frac{e E_{L}}{m c \omega_{0}}$ [36]. The $t_{0}$ can be considered to be the injection time $t_{0}=\frac{\gamma_{g}}{\omega_{\mathrm{p}} a_{0}}$. This time is not short; it is of the order of 1 ps even for this maximum plasma field. Moreover, an electron, appearing inside a laser pulse, is trapped by the laser pulse fields and is accelerated by the pulse for a rather long time, acquiring momentum opposite to the laser propagation direction owing to the ponderomotive force. The soft condition for the length results in a stronger condition for ionization injection on the laser pulse length and plasma density, $c \tau / 2+L<\lambda_{\mathrm{pl}} / 2$, where $\tau$ is the total pulse duration: Half of the laser pulse length plus the acceleration length should be shorter than the length of the acceleration phase of the wake wave (we assume here that the acceleration length equals approximately half of the wake wavelength). It is clear that ionization injection can occur for low plasma density and rather short laser pulses. This explains the similarity in the results of electron acceleration in pure $\mathrm{Ar}$ and $\mathrm{He}$ gases at high densities [37]. However, for lower plasma density, several groups have reported a clear dependence of the results on the dope quantity [28]. Nevertheless, there is an alternative point of view that the dope may aid in the modulation of plasma density by low-intensity laser prepulses [38,39], resulting in much better focusability of the laser pulses. All these facts require detailed analysis of 


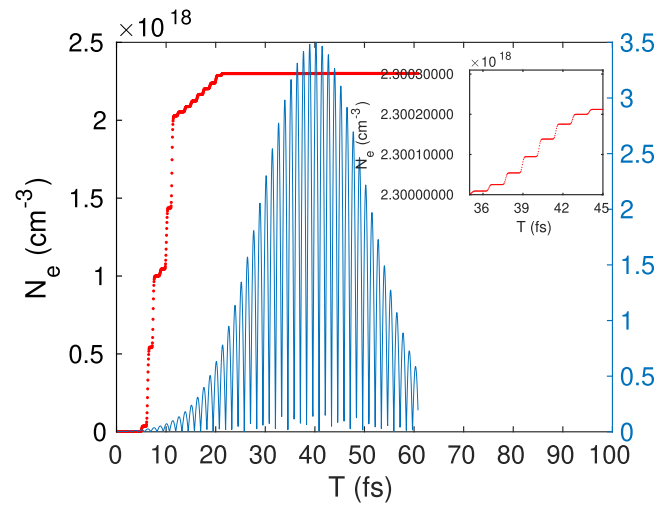

(a)

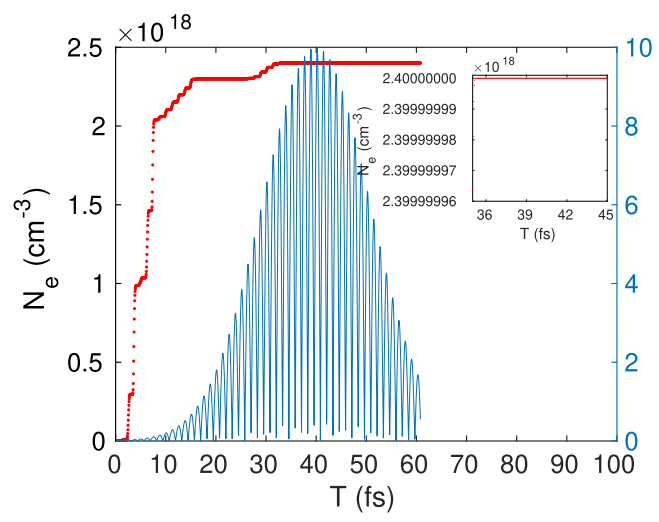

(c)

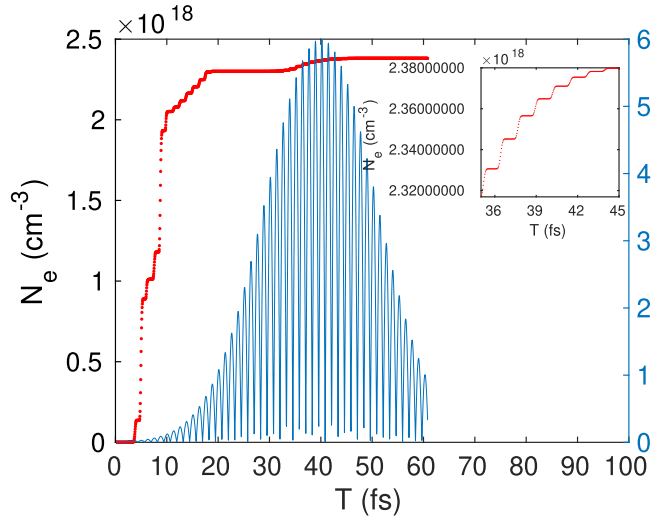

(b)

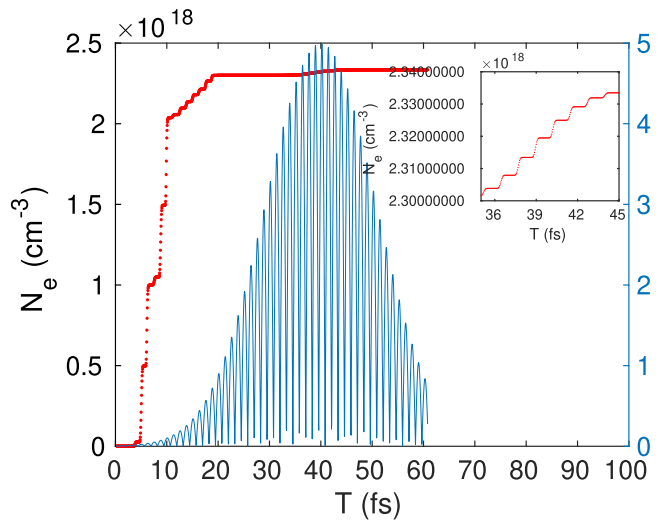

(d)

FIG. 2. Dynamics of the electron density in the gas mixture He-Ne with $10 \%$ of $\mathrm{Ne}$ (a)-(c) along the laser axis and (d) off-axis, depending on the laser pulse intensity (a) $a_{0 \max }=3.5$, (b) $a_{0 \max }=6$, and (c) and (d) $a_{0 \max }=10$. Insets show the electron densities around the peak of the laser pulse.

the ionization injection and its efficiency via particle-in-cell simulation.

There are two main approaches in the calculation of plasma ionization in particle-in-cell methods. The first is the variable particle weight (VPW) technique [40-42] in the VillasenorBuneman method and the second is the variable number of particles (VNP) technique [43-46]. The first is easier practically and less time consuming, while the second allows for satisfying the initial conditions for the particles when they are born. Not obeying the initial conditions for newly produced particles may sometimes result in nonphysical solutions (see, for example, Ref. [43]). Unfortunately, ionization injection also requires obeying the initial condition $p_{L}=0$, where $p_{L}$ is the particle momentum in the direction of the laser pulse propagation, and the VPW technique is not applicable in its simple form. However, the density of ionization electrons is usually small compared to the plasma density and the parameter $\eta / Z \ll 1$ even for $\eta=1$ (here $\eta$ is the doping ratio and $Z$ is the dope element charge). Direct use of the method of VNP [45] with the necessary accuracy becomes impractical also owing to a very small density increase after ionization of the inner shell. On the other hand, the low density of ionization electrons allows for the use of a perturbative approach in the solution of the problem of ionization injection. A combination of methods for background plasma electrons and for electrons appearing after inner shell ionization, for example, VNP plus VNP with different resolution, VPW plus VNP, no ionization plus VNP, and splitting electron particles into several groups, allows investigating the wave breaking injection and the ionization injection separately.

According to the calculations in Ref. [42], propagation of a laser pulse in high- $Z$ gas with optical field ionization runs similarly to that in a plasma without ionization if the plasma density is properly determined. Again, the doping of a high- $Z$ gas in a low- $Z$ gas can be considered as a small perturbation for the plasma and laser field. It is clear that for such conditions ionization injection can be characterized by a few parameters: plasma density, plasma density surplus in the vicinity of the laser pulse maximum, laser pulse intensity, and (weakly) the spatial distribution of the laser light. In the present paper analysis of ionization injection depending upon these parameters is performed in three parts. In the first part the kinetics of the ion states, including inner shells, is considered for $\mathrm{He}-\mathrm{N}_{2}$ and $\mathrm{He}-\mathrm{Ne}$ gas mixtures to understand the spatial distribution of the ionization electron density. The second part is devoted to the investigation of ionization electron trajectories in real wake fields depending on the initial position of the electrons. In the third part charge loading in the acceleration phase of laser pulse wake owing to the ionization injection for different doping 


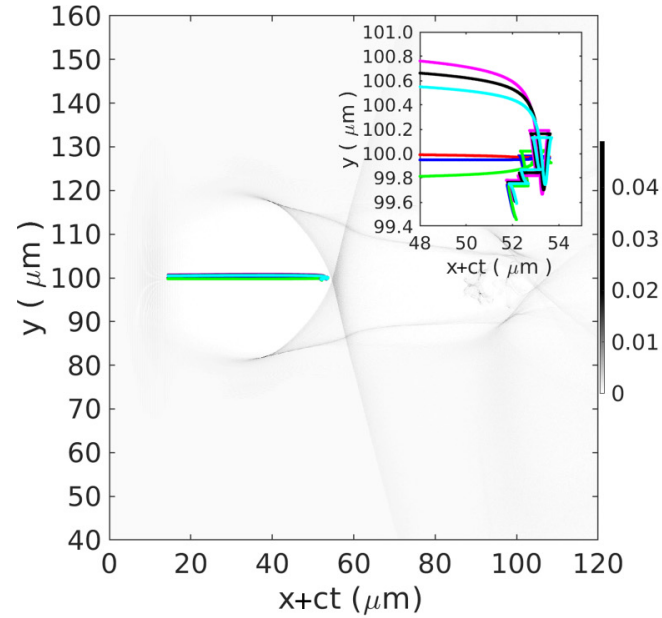

(a)

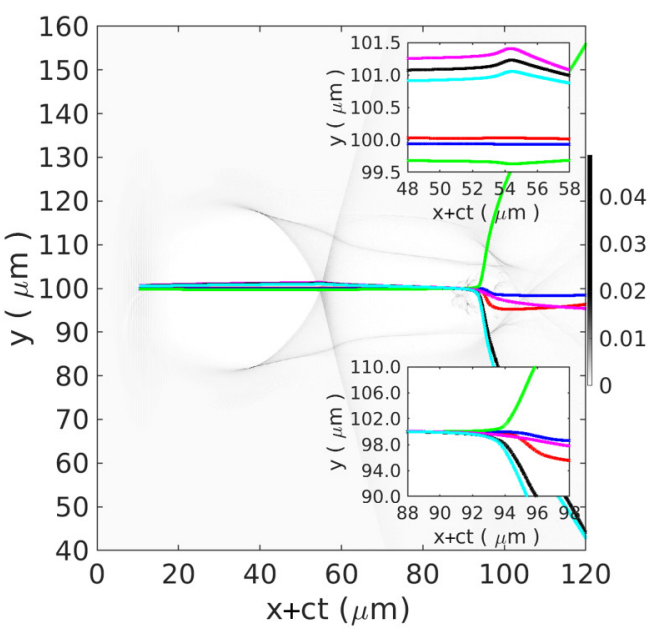

(c)

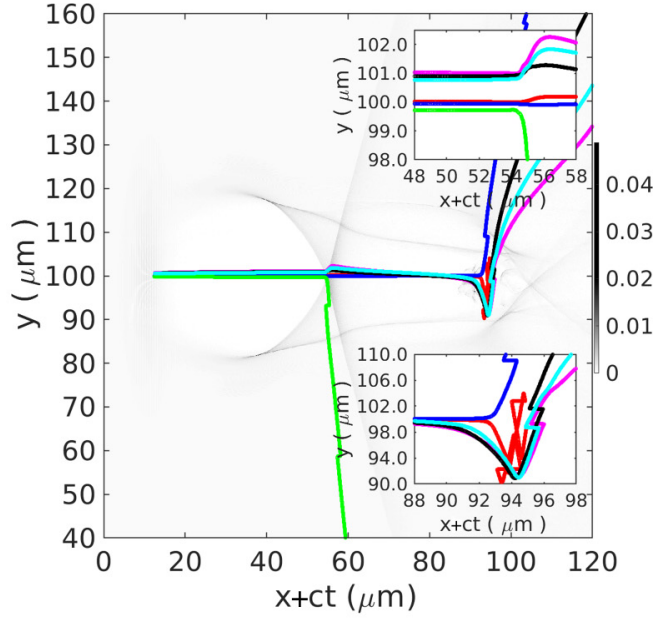

(b)

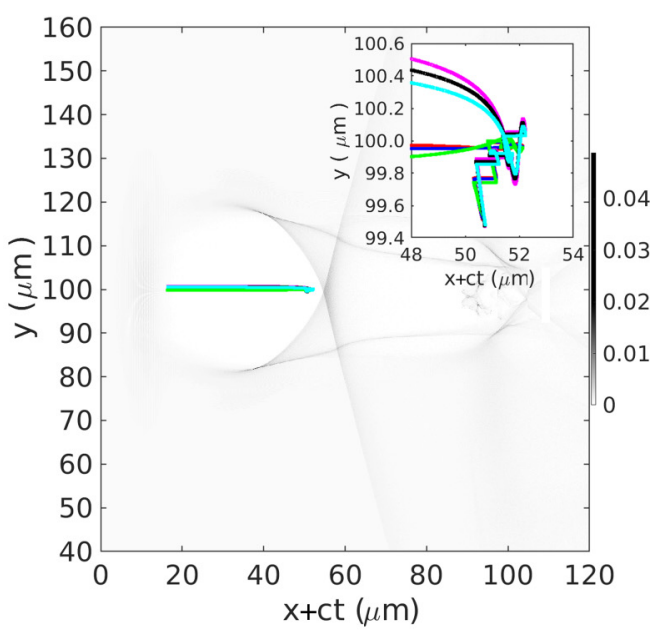

(d)

FIG. 3. Trajectories of test probe particles placed at distance $\Delta x$ from the maximum the laser pulse with 30 -fs duration and intensity $I=3 \times 10^{19} \mathrm{~W} \mathrm{~cm}^{-2}$ : (a) $\Delta x=0 \mu \mathrm{m}$, (b) $\Delta x=-2 \mu \mathrm{m}$, (c) $\Delta x=-4 \mu \mathrm{m}$, and (d) $\Delta x=2 \mu \mathrm{m}$. Different colors corresponds to different transverse positions of the probe particles: green, $\Delta y=-0.2 \mu \mathrm{m}$; blue, $\Delta y=-0.15 \mu \mathrm{m}$; red, $\Delta y=-0.1 \mu \mathrm{m}$; cyan, $\Delta y=0.5 \mu \mathrm{m}$; black, $\Delta y=0.6 \mu \mathrm{m}$; and magenta, $\Delta y=0.7 \mu \mathrm{m}$. Insets show a magnified view of different regions in the wave buckets. The laser pulse is propagating from right to left. The colorbar shows the electron density normalized by the critical density.

concentrations and plasma density is investigated via a selfconsistent particle-in-cell simulation with separated postprocessing for wave breaking and ionization electrons. This allows for an estimation of the efficiency of the different mechanisms of electron self-injection in gas mixtures.

\section{CHARGE STATES IN He-N 2 AND He-Ne MIXTURES}

The ion charge distribution under the action of strong femtosecond laser fields can be calculated with the common balance equations. In the absence of recombination, the set of equations is rather simple,

$$
\begin{aligned}
& \frac{\partial N_{0}}{\partial t}=-S_{0} N_{0}, \\
& \frac{\partial N_{z}}{\partial t}=S_{z-1} N_{z-1},
\end{aligned}
$$

and

$$
\frac{\partial N_{k}}{\partial t}=S_{k-1} N_{k-1}-S_{k} N_{k}, \quad k=1, \ldots, Z-1,
$$

where $N_{k}$ is the density of ions with charge $k, S_{k}$ is the optical field ionization rate, and $Z$ is the nuclear charge of an element. There are several approximations for the ionization rate [47]. We use the form

$$
S_{k}=4 \omega_{A} g_{k}\left(I_{k} / R y\right)^{5 / 2}\left(E_{A} / E_{L}\right) \exp \left[-\frac{2}{3}\left(I_{k} / R y\right)^{3 / 2}\left(E_{A} / E_{L}\right)\right]
$$

(in $\mathrm{s}^{-1}$ ), with ionization potentials $I_{k}$ for $\mathrm{He}, \mathrm{N}_{2}$, and $\mathrm{Ne}$ ions taking data from the most comprehensive calculations and experiments [48]. Here $E_{A}=m^{2} e^{5} / \hbar^{4}, \omega_{A}=m e^{4} / \hbar^{3}, R y=$ $m^{2} e^{4} / 2 \hbar^{2}, g_{k}$ is a factor of the order of unity, and $E_{L}$ is the laser field strength. For a gas mixture Eq. (2) should be calculated for each gas species. In Eq. (2) we implicitly assume that the density of ions with charge $n$ for which $\left(I_{n} / R y\right)^{3 / 2} E_{A} / E_{L} \ll 1$ is negligibly small. 


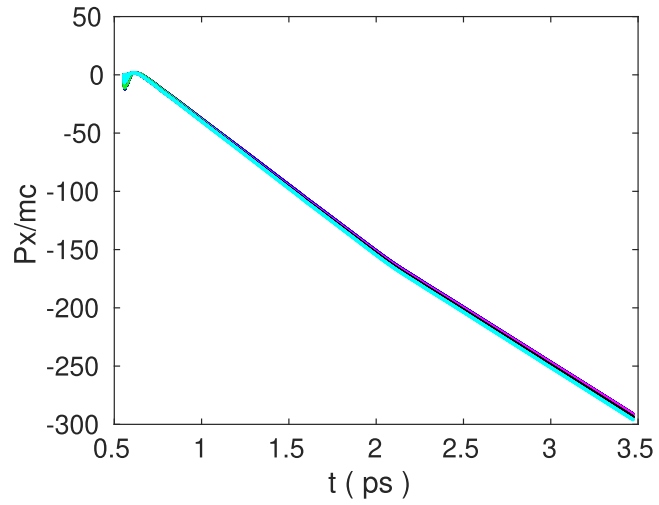

(a)

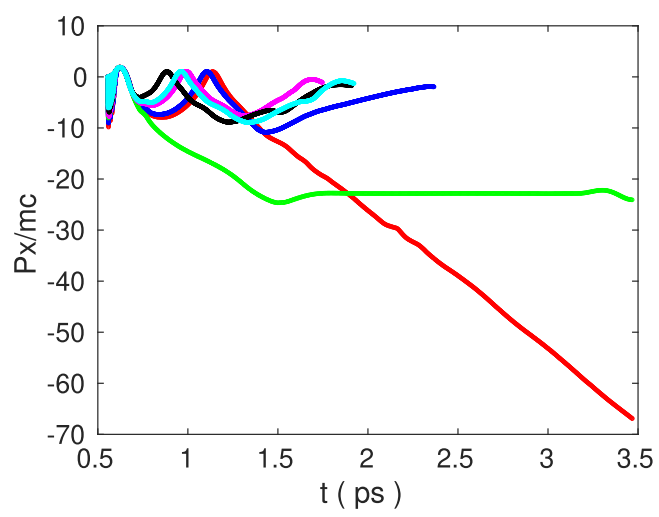

(c)

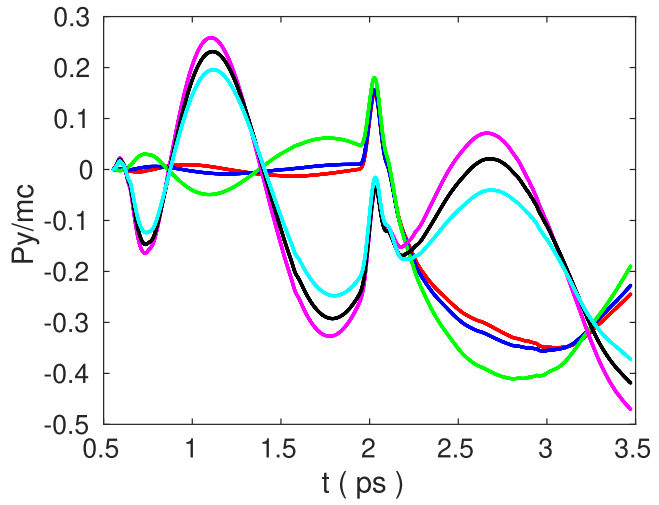

(b)

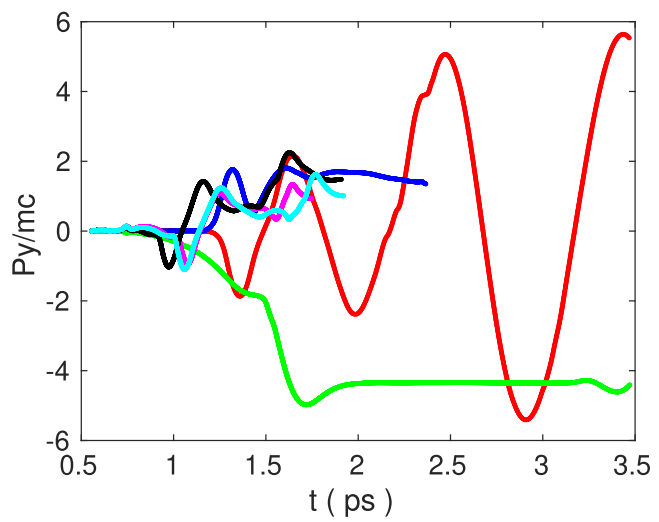

(d)

FIG. 4. Evolution of the longitudinal and transverse momenta of the probing particles placed at a distance $\Delta x$ from the laser pulse with 30-fs duration and intensity $I=3 \times 10^{19} \mathrm{~W} \mathrm{~cm}^{-2}$ : (a) and (b) $\Delta x=0 \mu \mathrm{m}$ and (c) and (d) $\Delta x=-2 \mu \mathrm{m}$. Different colors corresponds to different transverse positions of the probe particles: green, $\Delta y=-0.2 \mu \mathrm{m}$; blue, $\Delta y=-0.15 \mu \mathrm{m}$; red, $\Delta y=-0.1 \mu \mathrm{m}$; cyan, $\Delta y=0.5 \mu \mathrm{m}$; black, $\Delta y=0.6 \mu \mathrm{m}$; and magenta, $\Delta y=0.7 \mu \mathrm{m}$.

Numerical solution of Eq. (2) with the conservative condition

$$
\sum_{0}^{Z} N_{k}=\mathrm{const}
$$

is not difficult. It is clear that there is a saturation level of ion charge owing to the exponential dependence of the ionization rate on the ionization potential. In He gas such a saturation occurs at the front of a powerful laser pulse. In the presence of a high- $Z$ dope, ionization of the outer shells and inner shell of the ions is different because the ionization potential of the inner shell is essentially higher than those for the outer shells by the parameter $\xi=[2 Z /(Z-2)]^{2}$; for $Z \gg 1$ it becomes equal to 4 . This means that after a plateau there may be a density jump in the vicinity of the maximum of the laser pulse field strength.

In Figs. 1 and 2 typical distributions of the electron density produced by a Gaussian laser pulse with different intensities and a duration of $30 \mathrm{fs}$ (such a pulse duration is typical for most of the existing high-power laser facilities) are presented for $\mathrm{He}-\mathrm{N}_{2}$ and $\mathrm{He}-\mathrm{Ne}$ gas mixtures. To make the jump more visible, the concentrations of the dopes are chosen to be 5\% for $\mathrm{N}_{2}$ and $10 \%$ for Ne. Figures 1(a)-1(c) show the evolution of the electron density along the laser axis. In the case of the nitrogen dope essentially a density jump in the vicinity of the laser pulse maximum (clear in the insets) is seen for $a_{0}=1.5$ and vanishes for $a_{0}=3.5$. Within a distance of about $1 \mu \mathrm{m}$ near the pulse maximum, the density growth $\Delta N$ is less than $1 \%$ for all sets of $a_{0}$. With an increase of $a_{0}$, the density jump apparently shifts towards the front of the laser pulse. However, along the periphery of the laser pulse there is a density jump for $a_{0}=3.5$, as can be seen in Fig. 1(d). Such a jump has a ringlike shape. Further dynamics of such electrons have to be investigated as well. The higher ionization potential of Ne results in a higher $a_{0}$ necessary to have a density jump in the vicinity of the maximum laser pulse, as can be seen in Figs. 2(a)-2(c) (see the insets). In the case of $a_{0}=6$ the density growth reaches several percent and exceeds that for nitrogen. For $a_{0}>8$ the density jump vanishes in the vicinity of the laser pulse field maximum and shifts towards its front. This is important for further electron dynamics as described in the next Section. Again, there is a ring-shaped density jump in the periphery of the laser pulse even for $a_{0}=10$, as shown in Fig. 2(d).

The distributions of ionization electrons which can be extracted for Figs. 1 and 2 are very important in understanding the dynamics of ionization injection into the laser pulse 


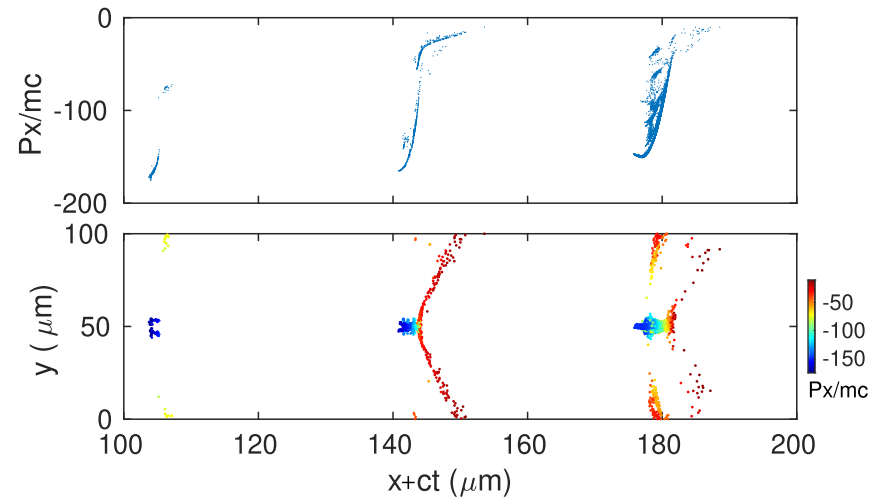

(a)

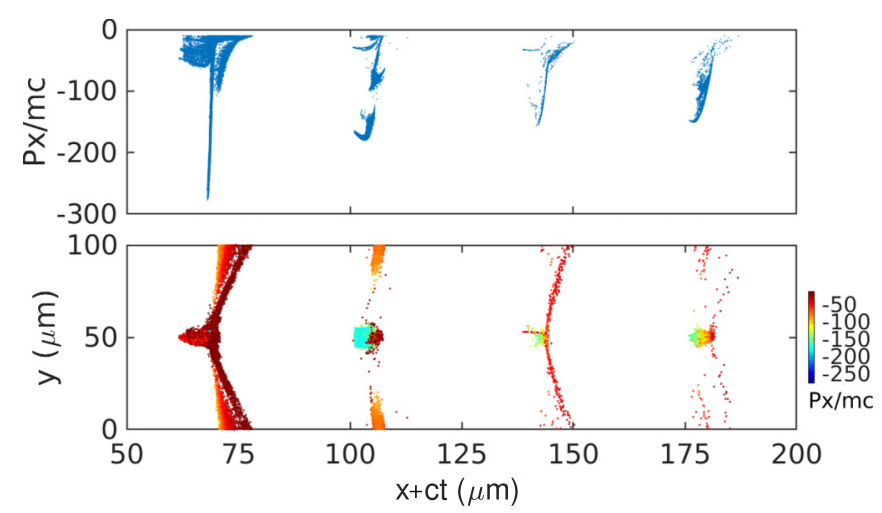

(b)

FIG. 5. Spatial distribution of the electrons' momenta at $t=$ $4.1 \mathrm{ps}$ in a plasma with $N_{e}=10^{18} \mathrm{~cm}^{-3}$ and $\Delta N=10^{16} \mathrm{~cm}^{-3}$ irradiated by a laser pulse with $I=3 \times 10^{19} \mathrm{~W} \mathrm{~cm}^{-2}$ and $\tau=30 \mathrm{fs}$ : (a) plasma electrons and (b) ionization electrons. The laser pulse is propagating from right to left.

wake. To clarify the physical picture of the dynamics of such electrons, we perform an investigation of electron trajectories in realistic laser wake fields.

\section{PROBING PARTICLES IN PARTICLE-IN-CELL SIMULATIONS}

To understand how the position of the ionization electron influences its further dynamics, we perform tests with probe particles during the propagation of a laser pulse in plasma using the particle-in-cell method. The simulations are performed in two-dimensional (2D) geometry, using the moving window technique. The window has size $100 \times 200 \mu \mathrm{m}^{2}$; the spatial grid resolution is $\lambda / 36$. Laser pulses with a duration of $30 \mathrm{fs}$ propagate in a uniform plasma with a density of $N_{e}=1 \times 10^{18} \mathrm{~cm}^{-3}$. The laser pulse intensity is varied from $I=1 \times 10^{19} \mathrm{~W} \mathrm{~cm}^{-2}$ to $I=3 \times 10^{19} \mathrm{~W} \mathrm{~cm}^{-2}$ and the laser spot size is $w_{0}=10 \mu \mathrm{m}$. Probe particles with small charge are placed near the maximum of the laser pulse after $140 \mu \mathrm{m}$ of pulse propagation and start moving. There are five sets of test probe particles, which are distributed around the peak of the laser pulse. Each set has six particles located at different positions in the transverse direction. Two sets of test particles are placed at the front of the laser pulse and two sets are

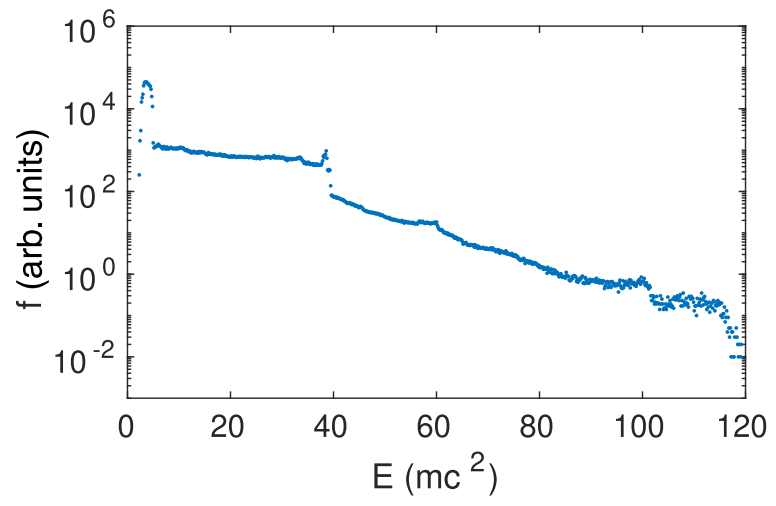

(a)

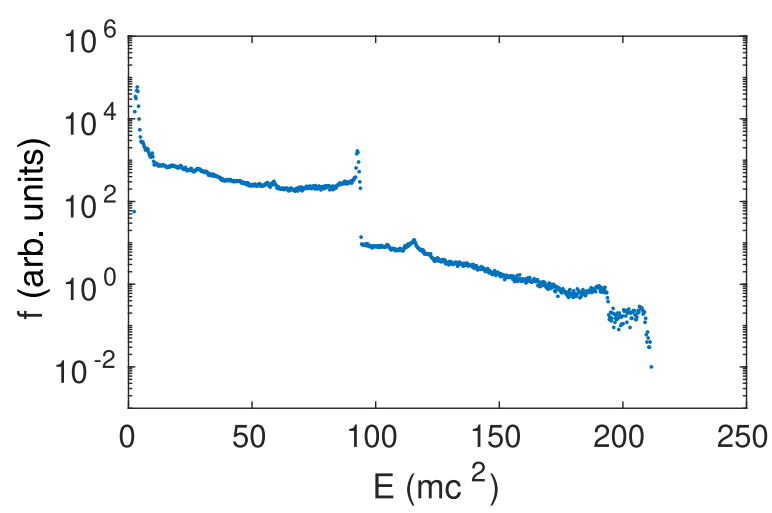

(b)

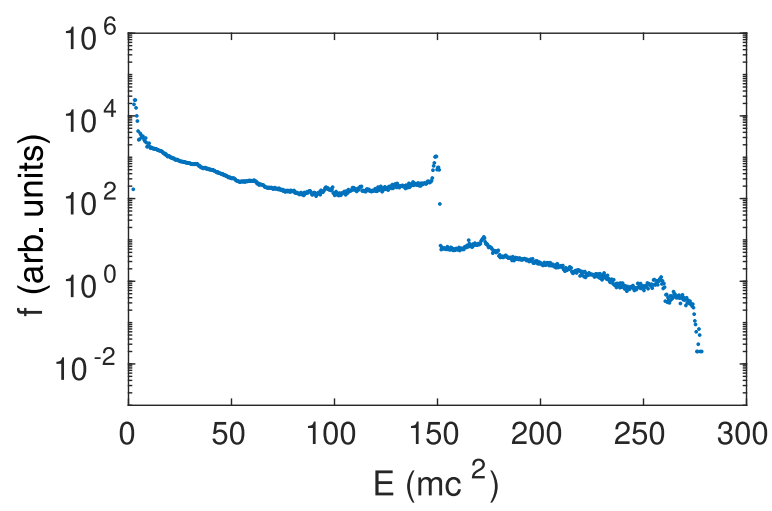

(c)

FIG. 6. Evolution of the total energy distribution for a plasma with $N_{e}=10^{18} \mathrm{~cm}^{-3}$ and $\triangle N=10^{16} \mathrm{~cm}^{-3}$ irradiated by a laser pulse with $I=3 \times 10^{19} \mathrm{~W} \mathrm{~cm}^{-2}$ and $\tau=30$ fs at (a) $t=1.7 \mathrm{ps}$, (b) $t=2.9 \mathrm{ps}$, and (c) $t=4.1 \mathrm{ps}$.

placed at the rear. One set of particles is placed at the peak of the pulse. These positions mimic well the appearance of ionization particles with initially zero momentum.

The resulting trajectories of the probe electrons for laser pulse intensity $I=3 \times 10^{19} \mathrm{~W} \mathrm{~cm}^{-2}$ are presented in Fig. 3 at $t=3.5 \mathrm{ps}$. Particles situated at the maximum of the laser pulse have the trajectories shown in Fig. 3(a). Initially particles are trapped by the laser pulse $v \times B$ force and move along the laser pulse propagation direction. Then the particles, having been overrun by the laser pulse, are taken up by the wake field 


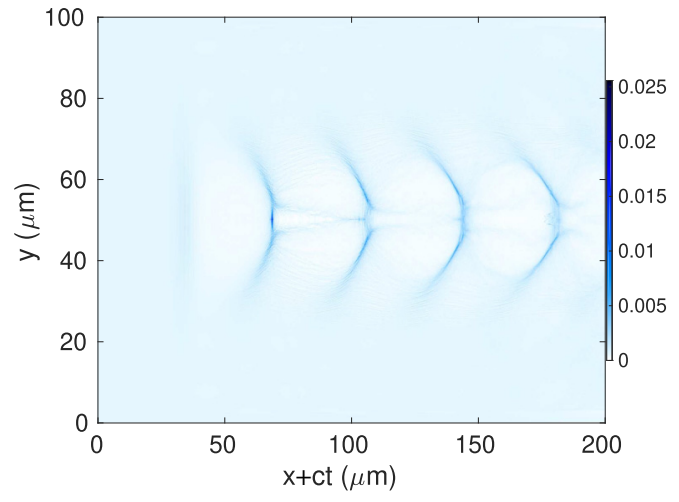

(a)

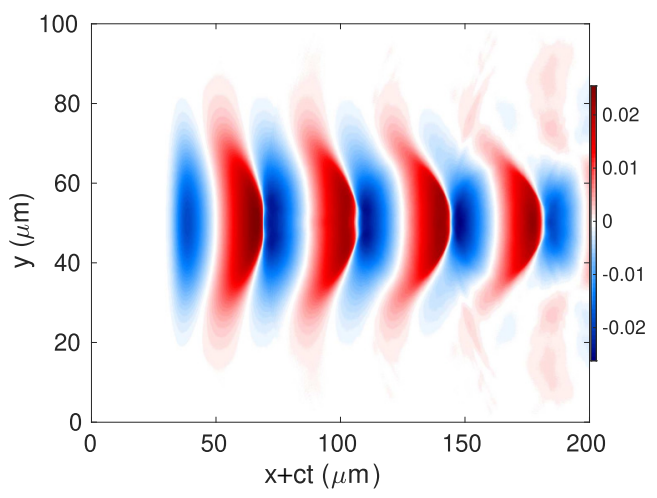

(b)

FIG. 7. Spatial distribution of (a) electron density and (b) $x$ component of the electric field for a plasma with $N_{e}=10^{18} \mathrm{~cm}^{-3}$ and $\triangle N=$ $10^{16} \mathrm{~cm}^{-3}$ irradiated by a laser pulse with $I=3 \times 10^{19} \mathrm{~W} \mathrm{~cm}^{-2}$ and $\tau=30 \mathrm{fs}$ at $t=4.1 \mathrm{ps}$. The laser pulse is propagating from right to left. The colorbar shows (a) the electron density normalized by critical density and (b) the axial field in normalized units $(e E x / m \omega c)$.

and accelerated. After approximately 1 ps all these particles are accelerated above the phase velocity of the plasma wave and therefore are injected. The evolution of their momenta can be seen in Fig. 4(a): After brief motion in the laser pulse the probe particles are accelerated to over $100 \mathrm{MeV}$ after several picoseconds. The relativistic factor for the phase velocity corresponding to the plasma density is $\gamma_{\mathrm{ph}}=\sqrt{\frac{N_{\mathrm{cr}} a_{0}}{N_{e}}} \approx 70$. The particles reach this energy after about 1.5 ps. The effect of side scattering by the ponderomotive force is illustrated in Fig. 4(b) by the transverse momenta of the particles. The effect exists but is small for the particles; the divergence for them is within $3 \times 10^{-3} \mathrm{rad}$.

A very different picture emerges in the case where the initial positions of the particles are before the maximum of the laser pulse field as shown in Fig. 3(b). One can observe no trapping of these particles in the first bucket of the wake. However, one particle is trapped and accelerated in the second bucket. The fact of trapping for this particle can be proved by the particle momentum shown in Fig. 4(c), where the velocity of this particle exceeds the phase velocity of the wave. The ratio of the transverse and longitudinal momenta for this particle is not small, of the order of 0.06. This shows that the divergence of a bunch formed by such particles should hardly be small. Particles situated farther from the pulse field maximum cannot be trapped even in the second bucket; a portion of them move to the third bucket and can be trapped there. This effect strongly depends on the longitudinal momentum acquired by the particle after the laser pulse has overrun it. The simulation shows that particles at the front of the laser pulse get a larger momentum directed counter to the laser pulse motion. Moreover, we observe no trapping in the first bucket of the probe particles situated at the maximum of the laser field in the case of lower pulse intensity $I=1 \times 10^{19} \mathrm{~W} \mathrm{~cm}^{-2}$ with the other conditions being the same. For higher intensity, some particles situated behind the maximum of the pulse field get almost zero momentum and can be efficiently trapped, as shown in Fig. 4(d). However, the number of such particles is not high, as can be seen in Figs. 1 and 2, where the increase in the number of ionization electrons after the peak of the laser pulse is small or negligible compared to the whole plasma density. In contrast, most of ionization electrons are born before the maximum of the laser field. This may result not only in continuous injection of ionization electrons in the first bucket but quite efficient injection of them into consequent buckets. Since the ionization injection is quite efficient, in the

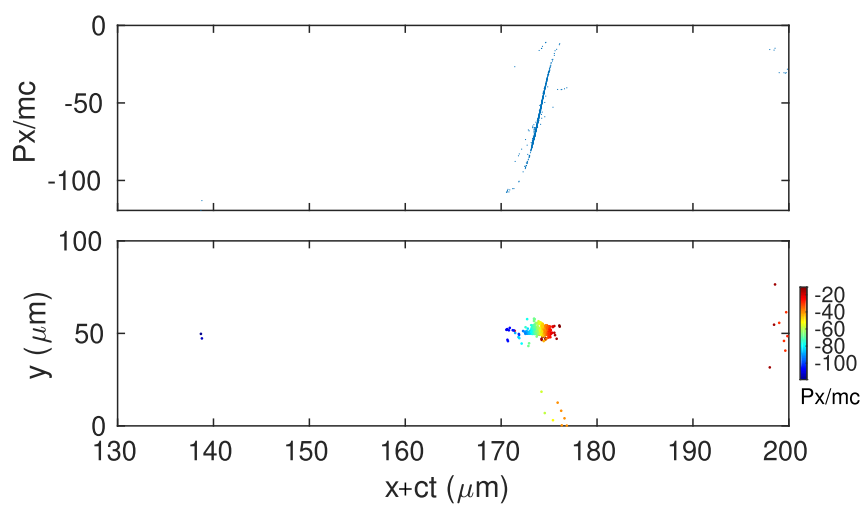

(a)

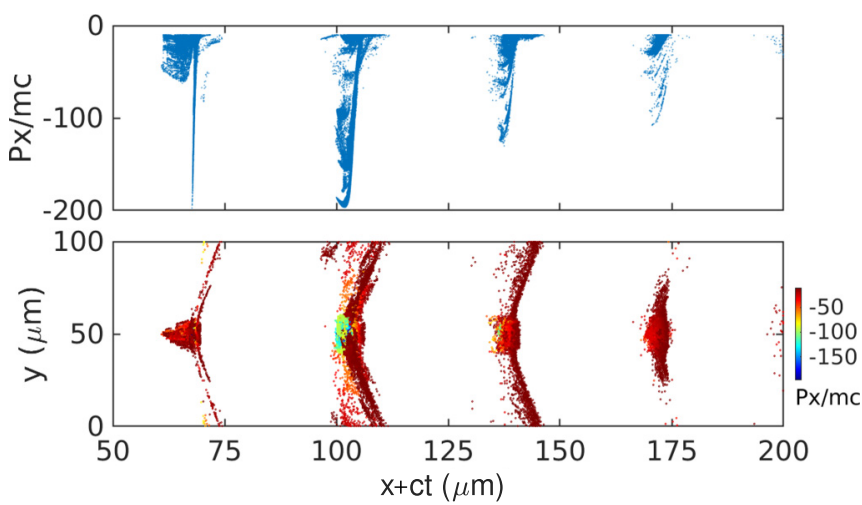

(b)

FIG. 8. Spatial distribution of the electrons' momenta at $t=$ $4.1 \mathrm{ps}$ in a plasma with $N_{e}=10^{18} \mathrm{~cm}^{-3}$ and $\triangle N=10^{17} \mathrm{~cm}^{-3}$ irradiated by a laser pulse with $I=3 \times 10^{19} \mathrm{~W} \mathrm{~cm}^{-2}$ and $\tau=30 \mathrm{fs}$ : (a) plasma electrons and (b) ionization electrons. The laser pulse is propagating from right to left. 


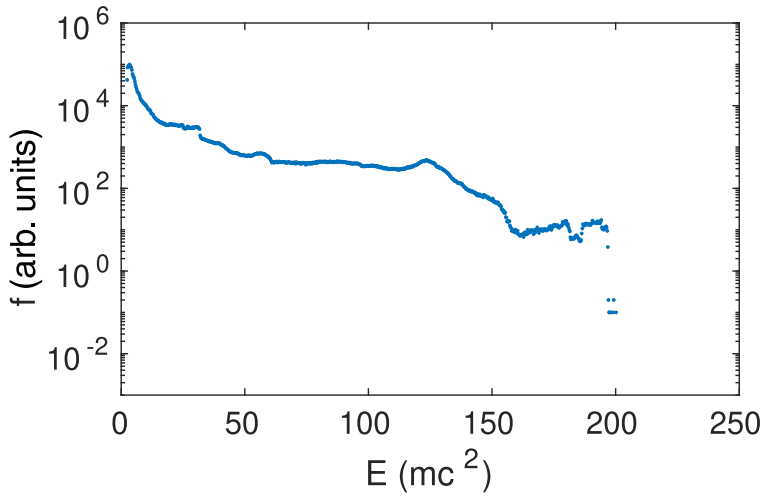

FIG. 9. Total energy distribution for a plasma with $N_{e}=$ $10^{18} \mathrm{~cm}^{-3}$ and $\triangle N=10^{17} \mathrm{~cm}^{-3}$ irradiated by a laser pulse with $I=3 \times 10^{19} \mathrm{~W} \mathrm{~cm}^{-2}$ and $\tau=30$ fs at $t=4.1 \mathrm{ps}$.

absence of wave breaking (no self-injection) the number of buckets can be large, which can result in a long length of the final electron bunches of the order of picoseconds.

\section{IONIZATION INJECTION IN LOW-DENSITY PLASMA}

The effects of ionization injection including charge loading can be calculated only with the use of the particle-in-cell method. It is apparent that ionization of plasma should be included. Upon considering a lower-intensity laser pulse $a_{0}<$ 10 we neglect ion motion and consider ionization processes using this approximation. Again, there are two methods to calculate plasma ionization in the framework of particle-incell simulation. The first is by the VPW method. In this method, particles emulating electrons are initially empty and are filled with electrons in time according to Eq. (1) [42]. This method is not time consuming; however, it has a disadvantage: It is impossible to apply arbitrary initial conditions for the electrons. For example, in the problem of light scattering from an ionization wave, the fact that the variable particle weight method results in unphysical solutions is explained in detail in [43]. Another method is the VNP [44-46], in which $N$ particles per cell have the same weight $W$. The value $N \times W$ represents the total possible electron density in the plasma. Initially, all particles are immobile. With time the number of movable particles increases in accordance with Eq. (1). A new particle is involved in the motion with the necessary initial conditions for its momentum. The disadvantage of this approach is that too many particles are necessary to make a smoother spatial electron density distribution. In the case of ionization injection, the density jump $\Delta N$ is rather small and the use of the VNP requires a resolution better than $\triangle N / N_{e}$ or a number of particles $N>\frac{N_{e}}{\Delta N}$.

There is an alternative approach allowing essential lessening of computing resources with a rather accurate evaluation of the ionization injection requiring rather high spatial resolution. The alternative is in the combination of the VNP and VPW techniques. For the case of $\Delta N / N_{e} \ll 1$, which is typical for experiments with doping, it is reasonable to use two groups of electrons, which are distinguishable from each other by a numerical parameter [see Fig. 1(b)]. The first one serves as plasma electrons. The dynamics of these

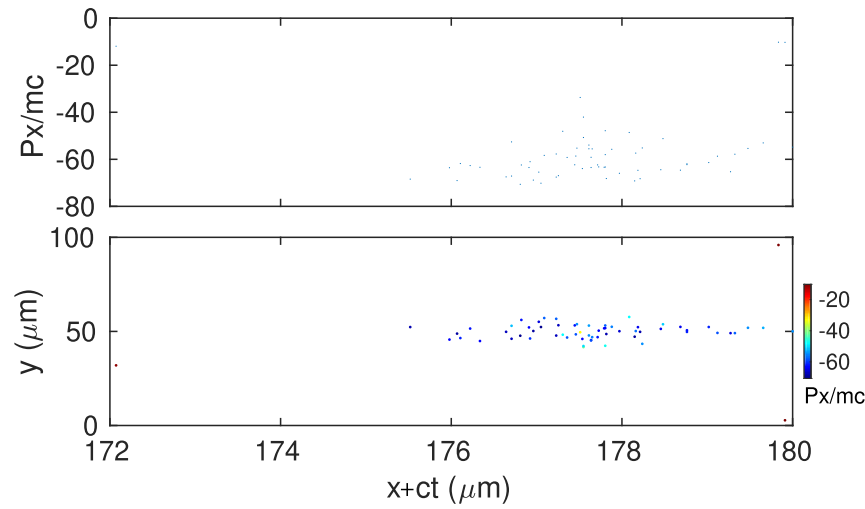

(a)

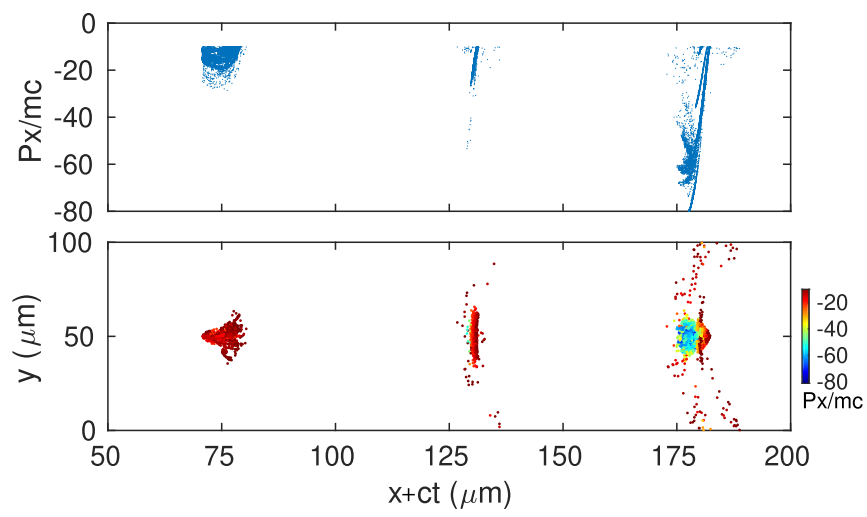

(b)

FIG. 10. Spatial distribution of the electrons' momenta at $t=$ $4.1 \mathrm{ps}$ in a plasma with $N_{e}=5 \times 10^{17} \mathrm{~cm}^{-3}$ and $\triangle N=5 \times 10^{16} \mathrm{~cm}^{-3}$ irradiated by a laser pulse with $I=3 \times 10^{19} \mathrm{~W} \mathrm{~cm}^{-2}$ and $\tau=30 \mathrm{fs}$ : (a) plasma electrons and (b) ionization electrons. The laser pulse is propagating from right to left.

electrons can be calculated both with the use of the VPW method or assuming a preionized plasma [42]. The second group consists of ionization electrons for which the VNP method is applied. We use for this group $m$ slices with charges proportional to $\Delta N / m$ and with a motion parameter which switches from false to true when a $k$ immovable ionization particle crosses (in the moving window) the vicinity of the laser field with strength $E_{L k}$. With the motion parameter true the ionization particle is involved in self-consistent motion similar to plasma particles. The simplest case is the single slice of ionization particles which start moving when crossing the maximal strength of the laser field. We perform such an alternative particle-in-cell simulations in two dimensions for gas mixtures using $N_{e}$ and $\Delta N$ as parameters for a plasma irradiated by a 30 -fs laser pulse with $\lambda=0.8 \mu \mathrm{m}$ and intensity $I=3 \times 10^{19} \mathrm{~W} \mathrm{~cm}^{-2}$ focused to $w_{0}=10 \mu \mathrm{m}$. The moving box has size $100 \times 200 \mu \mathrm{m}^{2}$ and a spatial grid resolution of $\lambda / 36$. The numbers of slices for ionization were 1 and 5 . We use a uniform plasma with a fixed density for easier control of the parameters. According to Ref. [42], such an approach does not essentially change the plasma field distributions even for pure high- $Z$ gas. The transverse distribution of the charges of the ionization particles is Gaussian with size equal $0.5 w_{0}$ centered around the laser propagation axis. 


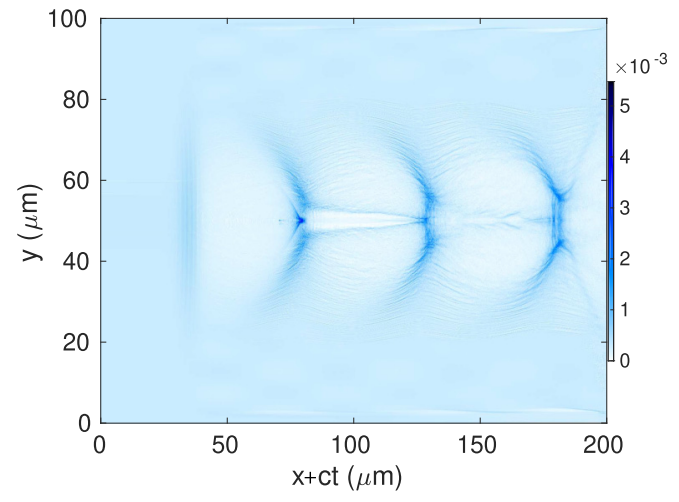

(a)

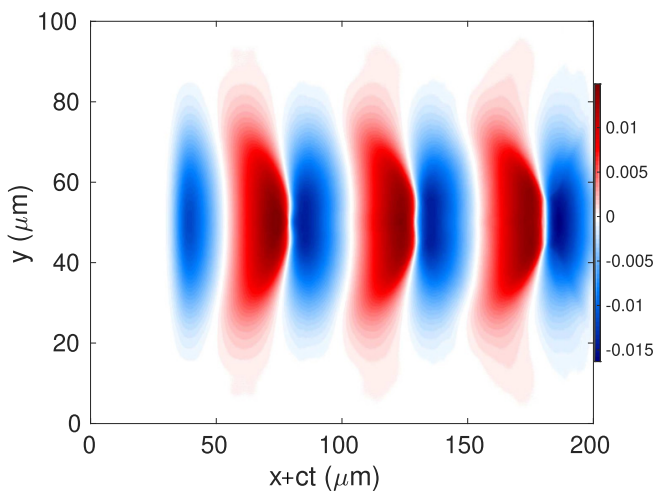

(b)

FIG. 11. Spatial distribution of (a) electron density and (b) $x$ component of the electric field for a plasma with $N_{e}=5 \times 10^{17} \mathrm{~cm}^{-3}$ and $\triangle N=5 \times 10^{16} \mathrm{~cm}^{-3}$ irradiated by a laser pulse with $I=3 \times 10^{19} \mathrm{~W} \mathrm{~cm}^{-2}$ and $\tau=30 \mathrm{fs}$ at $t=4.1 \mathrm{ps}$. The laser pulse is propagating from right to left. The colorbar shows (a) the electron density normalized by the critical density and (b) the axial field in normalized units $(e E x / m \omega c)$.

Momentum distributions for plasma and ionization particles for a plasma with density $N_{e}=1 \times 10^{18} \mathrm{~cm}^{-3}$ are shown in Fig. 5 at $t=4.1$ ps pulse propagation and for $\Delta N=$ $10^{-2} N_{e}$. Such a density jump corresponds to $5 \%$ of $\mathrm{N}_{2}$ according to the ionization balance in the laser pulse field. According to plasma particles [see Fig. 5(a)], the wave breaking injection is rather weak and occurs in the third and fourth buckets. As for ionization injection [Fig. 5(b)], it starts in the first bucket and gradually appears in the other buckets. The energy of the injected electrons in the first bucket reaches $150 \mathrm{MeV}$ after $4 \mathrm{ps}$ and increases. The electron energy in the second, third, and fourth buckets also increases similarly, with a clear time delay. The evolution of the total energy distribution taking into account the particle weights is shown in Figs. 6(a)-6(c). One can see two parts separated by a peak in the figures. The lower-energy part originates from the wave breaking, while the higher-energy part is from pure ionization injection. Both parts are flat without characteristic features. However, the wave breaking injection provides a larger charge of accelerated electrons than the ionization injection, as can be seen in Fig. 6(c). We also have to separate out different sorts of electrons from the ionization injection. There are many electrons involved in the initial acceleration by the pulse wake. These electrons acquire certain energies around up to tens of $\mathrm{MeV}$. However, they are not finally trapped in any bucket and form a sort of clouds with large divergence. The number of such electrons far exceeds the number of injected electrons. Another negative feature of ionization injection is the lengthening of the total electron bunch after electron injection in consequent buckets. Since the wave breaking process is not strong, as can be seen in Fig. 7, the number of buckets is quite large. All these buckets will be filled with injected electrons which are further accelerated. Such beams may have durations of several picoseconds.

The results presented in Figs. 5-7 have been obtained for a rather low concentration of high- $Z$ gas. Figure 8 illustrates what happens if the density of high- $Z$ gas is increased by factor of 10. One can see that the ionization injection in this case becomes dominant. Moreover, it suppresses the electron self-injection by the wave breaking process as shown in Fig. 8(a). However, the maximal energy of the accelerated electrons is lower than that for the lower concentration of high- $Z$ gas (see also Fig. 9). The most efficient injection and acceleration occur in the second bucket, which reflects upon the increase of the effect of the preaccelerated but not injected electrons on the acceleration process. The number of such electrons essentially increases. It is important to note that the practical realization of such conditions requires much higher laser pulse power. Since now the electron density is formed by a high- $Z$ gas, the power of the laser pulse should be equal to 2-3 times the critical power not for the maximum ionization plasma density but for the minimum, which is almost 10 times smaller. This is because, as the laser pulse focuses, the intensity is low enough so that initially the laser is only propagating in a singly ionized plasma. To ensure relativistic focusing, this requires higher laser power. For the present parameters $\mathrm{N}_{2}$ gas requires a density of $N_{e}=1 \times$ $10^{18} \mathrm{~cm}^{-3}$, for which the laser pulse power should be about petawatt levels. Otherwise the pulse diffraction will shorten the length of the higher-density plasma. Working with high power may be critical to the ionization injection; however, this may restrict the process by itself. For laser pulse intensities approaching $I=1 \times 10^{20} \mathrm{~W} \mathrm{~cm}^{-2}$, relativistic wave breaking [16], which occurs for $a_{0} \sqrt{2\left(\gamma_{\mathrm{ph}}-1\right)}$, along with transverse wave breaking [17], which occurs for $\lambda_{\mathrm{pl}} a^{1 / 2} / w_{0} \gg 1$, may make the wave breaking injection dominant even for lowdensity plasma.

The pure ionization injection case, which is at lower density than the previous cases, is illustrated in Fig. 10. In a plasma with density $N_{e}=5 \times 10^{17} \mathrm{~cm}^{-3}$ and $\triangle N=$ $5 \times 10^{16} \mathrm{~cm}^{-3}$ irradiated by laser pulses with intensity $I=$ $3 \times 10^{19} \mathrm{~W} \mathrm{~cm}^{2}$ we observe no results for the wave breaking processes. It is clearly seen in Fig. 10(a) where there are no visible self-injected plasma electrons. In contrast, one can see in Fig. 10(b) an efficient injection to the third bucket with continuous electron acceleration after 4 ps of laser pulse propagation. In the first and second buckets there are only preaccelerated electrons. At this time, $t=4.1 \mathrm{ps}$, it is impossible to say whether these electrons will be further accelerated or not. In Fig. 11 spatial distributions of the total electron density and plasma electric field are shown. One can see a small channel-like structure appearing along the laser axis. 


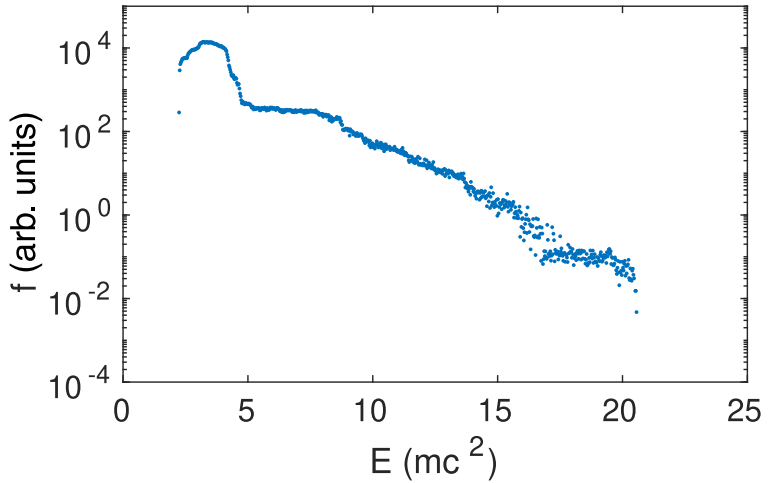

(a)

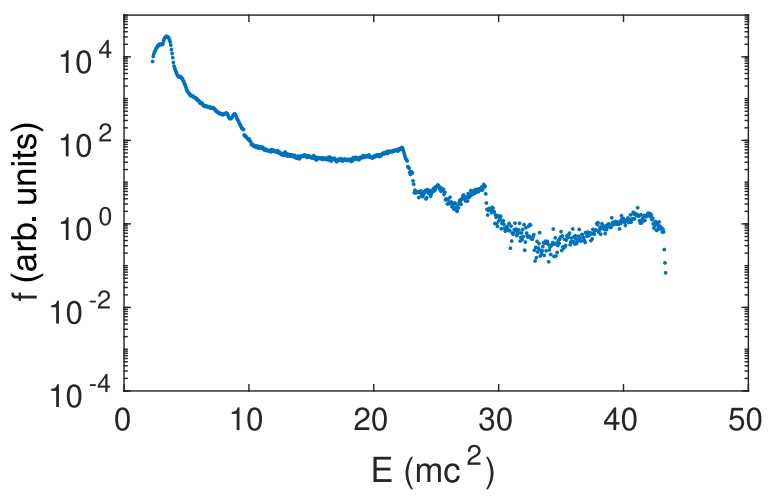

(b)

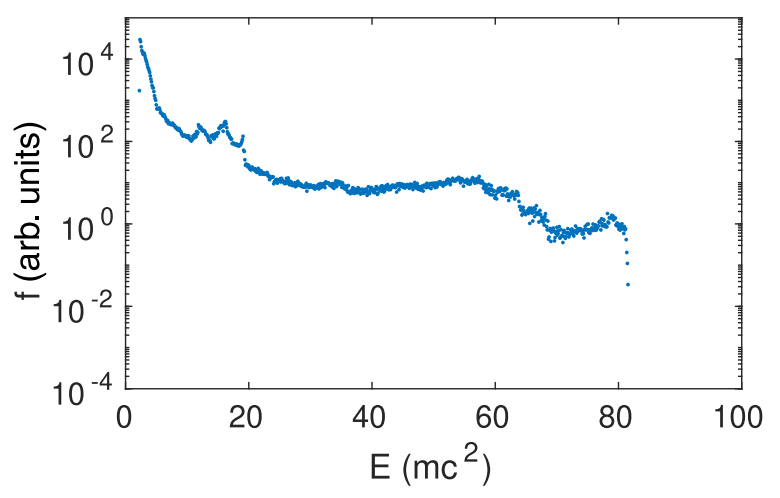

(c)

FIG. 12. Evolution of the total energy distribution for a plasma with $N_{e}=5 \times 10^{17} \mathrm{~cm}^{-3}$ and $\Delta N=5 \times 10^{16} \mathrm{~cm}^{-3}$ irradiated by a laser pulse with $I=3 \times 10^{19} \mathrm{~W} \mathrm{~cm}^{-2}$ and $\tau=30 \mathrm{fs}$ at (a) $t=1.7 \mathrm{ps}$, (b) $t=2.9 \mathrm{ps}$, and (c) $t=4.1 \mathrm{ps}$.

We anticipate that this structure is formed by preaccelerated electrons moving through the buckets. The evolution of the total distribution function is shown in Fig. 12. Even for $t=$ $4.1 \mathrm{ps}$ we observe no electrons with velocity exceeding the phase velocity of the plasma wave, which for this condition should be about $\gamma=100$.

\section{CONCLUSION}

We have characterized the effect of ionization injection for the laser wake field acceleration of electrons in gas mixtures using self-consistent particle-in-cell simulations. First, we applied the technique of test probe particles to investigate the condition of particle trapping in the acceleration phase of the wake field. Second, we performed two-dimensional particlein-cell simulations splitting plasma electrons and ionization electrons, which allowed us to investigate the entire process of electron preacceleration, trapping, and further acceleration.

Direct simulations have shown the efficiency of continuous ionization injection in a low-density plasma with low- and high- $Z$ components. Electrons, generated by optical field ionization of the ion inner shell in the vicinity of the maximum of the laser pulse field, after their propagation inside the pulse can be preaccelerated by the wake field to energies high enough for their further acceleration. These electrons can form a high-energy low-divergence beam. The total charge of the beam depends on the matching between the laser intensity and the high- $Z$ component. Electrons appearing before the maximum of the laser pulse field due to ionization of the inner shell cannot be trapped in the first bucket and finally form a cloud of preaccelerated electrons or are injected in wake buckets far behind the laser pulse. The latter process results in the formation of a long electron bunch with a duration of several picoseconds. Optimal matching can provide a density jump equal to $\eta N_{e} / Z$, with $\eta$ being the concentration of the high- $Z$ gas. With a plasma density increase the ionization injection cannot compete with the wave breaking injection. Moreover, when the laser pulse length exceeds the plasma wavelength, the ionization injection vanishes.

Typically in experiments the density of the high- $Z$ gas is small, being of the order of a few percent. For densities of ionization electrons $\Delta N=10^{15}-10^{16} \mathrm{~cm}^{-3}(5 \%-10 \%$ of the high- $Z$ dope) and a diameter of the injection section of about $5 \mu \mathrm{m}$ (see Figs. 1 and 2), one can estimate the total charge per millimeter of acceleration length to be $40-400 \mathrm{pC} / \mathrm{mm}$. However, the low efficiency for trapped electrons, which is less than $10 \%$ of the total preaccelerated particles, essentially reduces the charge of accelerated electrons. The total charge may be considerably higher after ionization injection fills many wake buckets. However, such beams can be quite long with their duration being picoseconds. Nevertheless, the ionization injection is a clear and important mechanism in the interaction of a short pulse laser with plasma which has to be taken into consideration in the development of, for example, plasma cathodes in the staging laser wake field acceleration. We recognize that the present research gives only a rough picture of this process, and the decision on its usefulness for a particular application should be done by particle-in-cell simulation based on the presented method. This examination has been done in a $2 \mathrm{D}$ approach. Differences in 2D and 3D particle-in-cell simulation results depends on the laser propagation regime. Two parameters that determine the propagation are $P / P_{\mathrm{cr}}$ and $w_{0}$, where $P$ is the laser pulse power, $P_{\mathrm{cr}}$ is the critical power for the self-focusing, and $w_{0}$ is the pulse waist. For $P / P_{\mathrm{cr}} \gg 1$, the self-focusing becomes strong and periodic. For the strong self-focusing the results of 2D and 3D geometries are numerically different [49] but qualitatively the same. In the case of moderate $P / P_{\mathrm{cr}}$, the most interesting for practical and reproducible ionization injection, the difference is not strong. Ionization injection in the case of strong self-focusing requires further analysis. 


\section{ACKNOWLEDGMENTS}

This work was funded by the JST-MIRAI program Grant No. JPMJMI17A1 and was partially supported by the ImPACT R\&D Program of Council for Science,
Technology and Innovation (Cabinet Office, Government of Japan). We are grateful to Prof. Yuji Sano for encouragement and helpful discussions. We also acknowledge the use of Mini-K computing facility at SACLA, RIKEN, SPring- 8 Center.
[1] T. Tajima and J. M. Dawson, Phys. Rev. Lett. 43, 267 (1979).

[2] S. P. D. Mangels, C. D. Murphy, Z. Najmudin, A. G. R. Thomas, J. L. Collier, A. E. Dangor, E. J. Divall, P. S. Foster, J. G. Gallacher, C. J. Hooker, D. A. Jaroszynski, A. J. Langley, W. B. Mori, P. A. Norreys, F. S. Tsung, R. Viskup, B. R. Walton, and K. Krushelnick, Nature (London) 431, 535 (2004).

[3] C. G. R. Geddes, C. Toth, J. van Tilborg, E. Esarey, C. B. Schroeder, D. Bruhwiler, C. Nieter, J. Cary, and W. P. Leemans, Nature (London) 431, 538 (2004).

[4] J. Faure, Y. Glinec, A. Pukhov, S. Kiselev, S. Gordienko, E. Lefebvre, J. P. Rousseau, F. Burgy, and V. Malka, Nature (London) 431, 541 (2004).

[5] W. P. Leemans, A. J. Gonsalves, H. S. Mao, K. Nakamura, C. Benedetti, C. B. Schroeder, C. Tóth, J. Daniels, D. E. Mittelberger, S. S. Bulanov, J. L. Vay, C. G. R. Geddes, and E. Esarey, Phys. Rev. Lett. 113, 245002 (2014).

[6] W. P. Leemans, B. Nagler, A. J. Gonsalves, C. Tóth, K. Nakamura, C. G. R. Geddes, E. Esarey, C. B. Schroeder, and S. M. Hooker, Nat. Phys. 2, 696 (2006).

[7] A. J. Gonsalves, K. Nakamura, J. Daniels, C. Benedetti, C. Pieronek, T. C. H. de Raadt, S. Steinke, J. H. Bin, S. S. Bulanov, J. van Tilborg et al., Phys. Rev. Lett. 122, 084801 (2019).

[8] A. Buck, J. Wenz, J. Xu, K. Khrennikov, K. Schmid, M. Heigoldt, J. M. Mikhailova, M. Geissler, B. Shen, F. Krausz, S. Karsch, and L. Veisz, Phys. Rev. Lett. 110, 185006 (2013).

[9] E. Esarey, C. B. Schroeder, and W. P. Leemans, Rev. Mod. Phys. 81, 1229 (2009).

[10] T. Ohkubo, S. V. Bulanov, A. G. Zhidkov, T. Esirkepov, J. Koga, M. Uesaka, and T. Tajima, Phys. Plasmas 13, 103101 (2006).

[11] G. Golovin, S. Banerjiee, S. Chen, N. Powers, C. Liu, W. Yan, J. Zhang, P. Zhao, and D. Umstadter, Nucl. Instrum. Methods Phys. Res. Sect. A 830, 375 (2009).

[12] Y. Sakai, N. Pathak, Z. Jin, A. Zhidkov, K. Sueda, H. Toran, Y. Tanizawa, T. Otsuka, J. Ogino, H. Nakamura, R. Kodama, and T. Hosokai, Phys. Rev. Accel. Beams 21, 101301 (2018).

[13] A. Oguchi, A. Zhidkov, K. Takano, E. Hotta, K. Nemoto, and K. Nakajima, Phys. Plasmas 15, 043102 (2008).

[14] V. B. Pathak, J. Vieira, R. A. Fonseca, and L. O. Silva, New J. Phys. 14, 023057 (2012).

[15] N. Pathak, A. Zhidkov, T. Hosokai, and R. Kodama, Phys. Plasmas 25, 013119 (2018).

[16] Z. M. Sheng and J. Meyer-ter-Vehn, Phys. Plasmas 4, 493 (1997).

[17] S. V. Bulanov, F. Pegoraro, A. M. Pukhov, and A. S. Sakharov, Phys. Rev. Lett. 78, 4205 (1997).

[18] J. Faure, C. Rechatin, A. Norin, A. Lifschitz, Y. Glinec, and V. Malka, Nature (London) 444, 737 (2006).

[19] S. Kalmykov, S. A. Yi, V. Khudik, and G. Shvets, Phys. Rev. Lett. 103, 135004 (2009).

[20] S. Corde, C. Thaury, A. Lifschitz, G. Lambert, K. Ta Phuoc, X. Davoine, R. Lehe, D. Douillet, A. Rousse, and V. Malka, Nat. Commun. 4, 1501 (2013).
[21] A. Pak, K. A. Marsh, S. F. Martins, W. Lu, W. B. Mori, and C. Joshi, Phys. Rev. Lett. 104, 025003 (2010).

[22] C. E. Clayton, J. E. Ralph, F. Albert, R. A. Fonseca, S. H. Glenzer, C. Joshi, W. Lu, K. A. Marsh, S. F. Martins, W. B. Mori, A. Pak, F. S. Tsung, B. B. Pollock, J. S. Ross, L. O. Silva, and D. H. Froula, Phys. Rev. Lett. 105, 105003 (2010).

[23] A. M. Ossa, T. J. Mehrling, L. Schaper, M. J. V. Streeter, and J. Osterhoff, Phys. Plasmas 22, 093107 (2015).

[24] J. P. Couperus, R. Pausch, A. Kohler, O. Zarini, J. M. Kramer, M. Garten, A. Huebl, R. Gebhardt, U. Helbig, S. Bock, K. Zeil, A. Debus, M. Bussmann, U. Schramm, and A. Irman, Nat. Commun. 8, 487 (2017).

[25] C. Kamperidis, V. Dimitrou, S. P. Mangles, A. E. Dangor, and Z. Najmudin, Plasma Phys. Control. Fusion 56, 084007 (2014).

[26] M. Zeng, M. Chen, Z. M. Sheng, W. Mori, and J. Zhang, Phys. Plasmas 21, 030701 (2014).

[27] M. Zheng, J. Luo, M. Chen, W. Mori, Z. M. Sheng, and B. Hidding, Phys. Plasmas 23, 063113 (2016).

[28] M. Mirzaie, S. Li, M. Zeng, N. A. M. Hafz, M. Chen, G. Y. Li, Q. J. Zhu, H. Liao, T. Sokollik, F. Liu, Y. Y. Ma, L. M. Chen, Z. M. Sheng, and J. Zhang, Sci. Rep. 5, 14659 (2015).

[29] C. Thaury, E. Guillaume, A. Lifschitz, K. Ta Phuoc, M. Hansson, G. Grittani, J. Gautier, J. P. Goddet, A. Tafzi, O. Lundh, and V. Malka, Sci. Rep. 5, 16310 (2015).

[30] P. Tomassini, S. Nicola, L. Labate, P. Londrillo, R. Fedele, D. Terzani, and L. A. Gizzi, Phys. Plasmas 24, 103120 (2017).

[31] Q. Zhao, S. M. Weng, Z. M. Sheng, M. Chen, G. B. Zhang, W. B. Mori, B. Hidding, D. A. Jaroszynski, and J. Zhang, New J. Phys. 20, 063031 (2018).

[32] M. Chen, E. Cormier-Michel, C. G. R. Geddes, D. L. Bruhwiler, L. L. Yu, E. Esarey, C. B. Schroeder, and W. P. Leemans, J. Comput. Phys. 236, 220 (2013).

[33] M. Chen, E. Esarey, C. B. Schroeder, C. G. R. Geddes, and W. P. Leemans, Phys. Plasmas 19, 033101 (2012).

[34] M. Chen, Z. M. Sheng, Y. Y. Ma, and J. Zhang, J. Appl. Phys. 99, 056109 (2006).

[35] C. McGuffey, A. G. R. Thomas, W. Schumaker, T. Matsuoka, V. Chvykov, F. J. Dollar, G. Kalintchenko, V. Yanovsky, A. Maksimchuk, K. Krushelnick, V. Y. Bychenkov, I. V. Glazyrin, and A. V. Karpeev, Phys. Rev. Lett. 104, 025004 (2010).

[36] E. Esarey, P. Sprangle, J. Krall, and A. Ting, IEEE Trans. Plasma Sci. 24, 252 (1996).

[37] M. Kando, S. Masuda, A. Zhidkov, A. Yamazaki, H. Kotaki, S. Kondo, T. Homma, S. Kanazawa, K. Nakajima, Y. Hayashi et al., Phys. Rev. E 71, 015403(R) (2005).

[38] T. Hosokai, K. Kinoshita, T. Ohkubo, A. Maekawa, M. Uesaka, A. Zhidkov, A. Yamazaki, H. Kotaki, M. Kando, K. Nakajima, S. V. Bulanov, P. Tomassini, A. Giulietti, and D. Giulietti, Phys. Rev. E 73, 036407 (2006).

[39] N. Lemos, L. Cardoso, J. Geada, G. Figueira, F. Albert, and J. M. Dias, Sci. Rep. 8, 3165 (2018) 
[40] A. Zhidkov and A. Sasaki, Phys. Plasmas 7, 1341 (2000).

[41] A. Zhidkov, A. Sasaki, and T. Tajima, Phys. Rev. E 61, R2224(R) (2000).

[42] K. Makito, A. Zhidkov, T. Hosokai, J. Shin, S. Masuda, and R. Kodama, Phys. Plasmas 19, 103104 (2012).

[43] A. Zhidkov, T. Esirkepov, T. Fujii, K. Nemoto, J. Koga, and S. V. Bulanov, Phys. Rev. Lett. 103, 215003 (2009).

[44] S. Kato, Y. Kishimoto, and J. Koga, Phys. Plasmas 5, 292 (1998).
[45] A. Zhidkov, J. Koga, T. Esirkepov, T. Hosokai, M. Uesaka, and T. Tajima, Phys. Rev. E 69, 066408 (2004).

[46] R. Nuter, L. Gremillet, E. Lefebvre, A. Levy, T. Ceccotti, and P. Martin, Phys. Plasmas 18, 033107 (2011).

[47] S. V. Popruzhenko, V. D. Mur, V. S. Popov, and D. Bauer, Phys. Rev. Lett. 101, 193003 (2008).

[48] NIST Atomic Spectra Database Ionization Energies Form, https://physics.nist.gov/PhysRefData/ASD/ionEnergy.html.

[49] A. A. Golovanov and I. Y. Kostyukov, Phys. Plasmas 25, 103107 (2018). 